\title{
Parameter Optimization for Transitions between Memory States in Small Arrays of Josephson Junctions
}

\author{
J. D. Rezac ${ }^{(1,4)}$, N. Imam ${ }^{(2)}$, and Y. Braiman ${ }^{(1,3)}$ \\ ${ }^{(1)}$ Computer Science and Mathematics Division \\ Computing and Computational Sciences Directorate \\ Oak Ridge National Laboratory \\ Oak Ridge, TN 37831 \\ ${ }^{(2)}$ Computing and Computational Sciences Directorate \\ Oak Ridge National Laboratory \\ Oak Ridge, TN 37831 \\ ${ }^{(3)}$ Department of Mechanical, Aerospace, and Biomedical Engineering \\ University of Tennessee \\ Knoxville, TN 37996 \\ ${ }^{(4)}$ Department of Mathematical Sciences \\ University of Delaware \\ Newark, DE 19716
}

\begin{abstract}
Coupled arrays of Josephson junctions possess multiple stable zero voltage states. Such states can store information and consequently can be utilized for cryogenic memory applications. Basic memory operations can be implemented by sending a pulse to one of the junctions and studying transitions between the states. In order to be suitable for memory operations, such transitions between the states have to be fast and energy efficient. In this paper we employed simulated annealing, a stochastic optimization algorithm, to study parameter optimization of array parameters which minimizes times and energies of transitions between specifically chosen states that can be utilized for memory operations (Read, Write, and Reset). Simulation results show that such transitions occur with access times on the order of $30-100$ ps and access energies on the order of $10^{-19}-5 \times 10^{-18} \mathrm{~J}$. Numerical simulations are validated with approximate analytical results.
\end{abstract}

PACS: 05.45.-a, 05.45.Xt, 85.25.Cp, 85.25.Hv

\section{Introduction}

Developing energy efficient and fast-access memory remains one of the main challenges in future computing systems. It may be possible to decrease memory access times and also reduce power consumption by utilizing cryogenic computing [1]. Cryogenic electronics based on superconducting devices (such as Josephson junctions, SQUIDS, etc.) are very fast and energy efficient [2]. A single Josephson junction can operate at a speed close to a $\mathrm{THz}$, and switching between the states may require as approximately $10^{-18}-10^{-19} \mathrm{~J}$.

Cryogenic memory plays an important role in the development of superconducting-based computing. A variety of designs have been proposed including memories based on single flux quantum (SFQ) digital logic [3], hybrid superconducting-CMOS designs [4, 5], magnetic random access memory (RAMs) [6], and others [7]. Some of the main challenges in developing superconducting memory are reducing power dissipation, increasing access speed, and reducing the size of the chip [7].

Recently we introduced a simple memory paradigm based on the existence of multiple stable states present in a large variety of nonlinear systems $[8,9]$. The transitions between those states act as basic memory cell operations (Write, Read, and Reset). The paradigm presented in references 8 and 9 is 
fundamentally different from the conventional paradigm that employs SFQ for memory operation. As an example, we presented the principles of operation of a circuit consisting of three inductively coupled Josephson junctions by implementing a mathematical model. In our design, an inductively coupled array of three Josephson junctions operates at cryogenic temperatures and is compact, fast, and energy-efficient. Write, Read, and Reset operations are applied to the same circuit to conserve area and decrease latency. In this paper, we describe the way in which these circuit parameter values affect the operation of the cryogenic memory circuit described in $[8,9]$ and optimize these values to improve memory operation. Because this circuit operates in a highly nonlinear regime, small perturbations to circuit parameters can affect circuit performance and functionality. In addition, fabrication imperfections are expected to lead to fluctuations in circuit parameters and circuit noise (e.g., pulse current noise or external drive noise) can also affect circuit performance. As such, we must be careful to ensure that memory cell operation operations are unaffected by such errors. To design an effective optimization procedure we ensure that: (a) small variation in circuit parameters and external pulse parameters do not affect the sequence and robustness of memory operations, and (b) small changes in circuit parameters and external pulse parameters do not result in significant changes in major characteristics of memory operations such as Read, Write, and Reset. Within the limits of (a) and (b), we are interested in choosing circuit parameters and external pulse parameters that will result in minimal memory access times and access energies.

In this Introduction section we review the critical components of the circuit design, define the values which are to be optimized, give a brief overview of the optimization results, and describe the method used to perform optimization studies. We also briefly review our circuit design, referring the reader to our papers $[8,9]$ for further details. In the following sections, we describe the results of the optimization procedure and show that our results are relatively impervious to noise.

\section{Overview of circuit design}

The memory logics under consideration are based on the transitions between pre-selected voltage states of a coupled array of Josephson junctions. The inductively coupled three-junction array is modeled by a nondimensional system of nonlinear equations (called as a resistively shunted junction, or RSJ, model),

$$
\begin{aligned}
& \frac{d^{2} \phi_{1}}{d \tau^{2}}+\gamma_{1} \frac{d \phi_{1}}{d \tau}+\sin \phi_{1}=i_{1}(\tau)+\kappa_{1}\left(\phi_{2}-\phi_{1}\right) \\
& \frac{d^{2} \phi_{2}}{d \tau^{2}}+\gamma_{2} \frac{d \phi_{2}}{d \tau}+\sin \phi_{2}=i_{2}(\tau)+\kappa_{1}\left(\phi_{1}-\phi_{2}\right)+\kappa_{2}\left(\phi_{3}-\phi_{2}\right) \\
& \frac{d^{2} \phi_{3}}{d \tau^{2}}+\gamma_{3} \frac{d \phi_{3}}{d \tau}+\sin \phi_{3}=i_{3}(\tau)+\kappa_{2}\left(\phi_{2}-\phi_{3}\right),
\end{aligned}
$$

Here, $\phi_{j}(\tau)$ is the phase of the junction as a function of dimensionless time $\tau$. The quantities $i_{j}(\tau)$ are currents applied to the circuit as a function of time. The parameters $\gamma_{j}$ are related to the resistance, current, and capacitance of the circuit and $\kappa_{j}$ are related to the strength of coupling in the circuit. Throughout the paper, we have fixed the values of $\gamma=0.7$ for the first and third junctions and $\gamma=1.1$ for the middle second junction. In the case of one array,

$$
\gamma=\sqrt{\frac{\hbar C}{2 e I_{c}}} \frac{1}{R C}=\left(\omega_{J} R C\right)^{-1}, \tau=\omega_{J} t, \omega_{J}^{2}=\frac{2 e}{\hbar} \frac{I_{c}}{C}, \quad \frac{I_{e x t}}{I_{c}}=i, \quad \text { and } \kappa=\frac{\Phi_{0}}{2 \pi L I_{c}},
$$

where $\Phi_{0}=\frac{h}{2 e}$ is the magnetic flux quantum, $h$ is Planck's constant, $e$ is electron charge, $R$ and $C$ are resistance and capacitance in the circuit, respectively, $L$ is the inductance of the inductor that couples Josephson junctions, $\tau$ is dimensional time, $I_{e x t}$ is external current, and $I_{c}$ is a critical current value related 
to non-dimensionalization. The relationships are similar for multiple arrays. In this paper we assume each $i_{j}(\tau)$ consists of a DC component and a Gaussian AC component so that $i_{j}(\tau)=i_{D C, \mathrm{j}}+\frac{i_{\text {pulse }, j}}{\sqrt{2 \pi \sigma_{j}^{2}}} e^{\frac{-\left(\tau-\tau_{j}\right)^{2}}{2 \sigma_{j}^{2}}}, j=1,2,3$ where $i_{D C, j}$ are the DC components, $i_{p u l s e, j}$ are related to the amplitude of the Gaussian pulse, and $\sigma_{j}$ are related to the width of the circuit. The parameter $\tau_{j}$ is a time-lag. Sometimes we use the notation $i_{A C, j}=\frac{i_{p u l s e, j}}{\sqrt{2 \sigma_{k}^{2}}}$ to refer to the maximum size of the AC pulse.

Typically, an RSJ model equation describing the dynamics of a single Josephson junction is written as $\beta_{c} \frac{d^{2} \phi}{d \tau^{2}}+\frac{d \phi}{d \tau}+\sin \phi=i$ in contrast to Equation (1) where $\gamma=1 / \sqrt{\beta_{c}}$. Consequently, the value of $\gamma=$ 0.7 corresponds to $\beta_{c} \approx 1.2$ which results in approximately $670-770 \mathrm{~nm}$ feature size junctions with the critical current density of $J_{c} \approx 50 \mathrm{kA} / \mathrm{cm}^{2}$ process [10].

\section{Circuit operation}

Key to the proposed Josephson junction circuit is the existence of distinct and well-defined voltage states which correspond to ' 0 ' and ' 1 ' memory states. These must be accessible from an applied trigger pulse. In this manuscript, Read, Write, and Reset operations are produced by applying a Gaussian pulse to a specified junction when the array is in steady state. For example, to write a ' 1 ,' an applied Gaussian pulse must have sufficient strength to transition the array from state ' 0 ' to state ' 1 .' However, this pulse must not be so strong to affect an array which is already in state ' 1 .'

The steady state phase values for the Josephson array can be characterized by a set of phases $\left\{\varphi_{1}, \varphi_{2}, \varphi_{3}\right\}=\left\{2 n_{1} \pi, 2 n_{2} \pi, 2 n_{3} \pi\right\}$ where $n_{j}$ are integers. As such, we refer to particular steady states by the set of integers $\left\{n_{1}, n_{2}, n_{3}\right\}$. Figure 1 shows the values of $i_{D C, 1}$ so that various steady states exist for a pre-defined parameter set given in the caption. As Figure 1 shows, when $i_{D C, 1}=1.0$, both states $\{0,0,0\}$ and $\{1,1,0\}$ are distinctive and there is a large gap between themselves and any other steady state. As such, for these values of $\kappa_{j}$ and $i_{D C, j}$, states $\{0,0,0\}$ and $\{1,1,0\}$ provide robust values for ' 0 ' and ' 1 ' states. In particular, we let $\{0,0,0\}$ correspond to ' 0 ' and $\{1,1,0\}$ to ' 1 .'

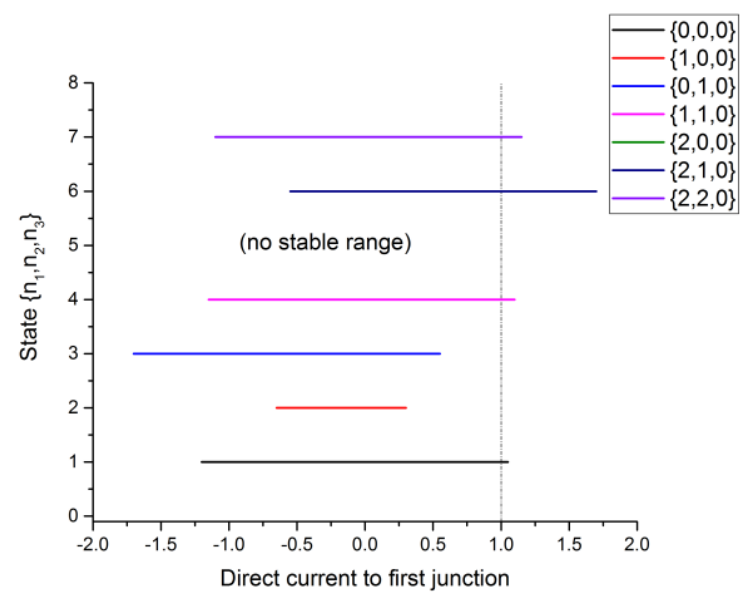

Figure 1: The ranges of stability for the available states as a function of $i_{D C, 1}$. In the Figure, $\kappa_{1}=\kappa_{2}=0.1, i_{D C, 2}=0.8, \gamma_{1}=$ $\gamma_{3}=0.7, \gamma_{2}=1.1$, and $i_{D C, 3}=-1.0$ in dimensionless units. The vertical dashed line shows $i_{D C, 1}=1.0$ which is typical of the values used in this paper.

A description of the way external pulses can move between memory states is given in Table 1. Full details about circuit operation can be found in $[8,9]$. 


\begin{tabular}{|c|c|c|c|}
\hline & Write '0' & Write '1' & Read \\
\hline $\begin{array}{c}\text { Original state } \\
\text { '0' }\end{array}$ & $\begin{array}{l}\text { Applied junction: } 3 \\
\text { End state: '0' } \\
\text { Read junction: } 3\end{array}$ & $\begin{array}{l}\text { Applied junction: } 1 \\
\text { End state: ' } 1 \text { ' } \\
\text { Read junction: } 2\end{array}$ & $\begin{array}{l}\text { Applied junction: } 1 \\
\text { End state: ' } 1 \text { ' } \\
\text { Read junction: } 2\end{array}$ \\
\hline $\begin{array}{c}\text { Original state } \\
' 1,\end{array}$ & $\begin{array}{l}\text { Applied junction: } 3 \\
\text { End State: '0' } \\
\text { Read junction: } 3\end{array}$ & $\begin{array}{l}\text { Applied junction: } 1 \\
\text { End state: ' } 1 \text { ' } \\
\text { Read junction: } 2\end{array}$ & $\begin{array}{l}\text { Applied junction: } 1 \\
\text { End state: ' } 1 \text { ' } \\
\text { Read junction: } 2\end{array}$ \\
\hline
\end{tabular}

Table 1: Description of the operations of the proposed memory device: Applied junction is the junction to which a Gaussian pulse is applied and read junction is the junction from which voltage is read to confirm operation has taken place. Original state is the initial state which the junction is in before the application of a pulse and end state is the final state the junction is after the application of a pulse.

\section{Optimization procedure}

Simulated Annealing (SA) is an iterative optimization algorithm which searches for global minima or maxima of given cost functions. SA explores the target function's entire surface by performing random walks in $M$ parameter space where $M$ represents the number of optimization variables. A rough view of the parameter space is first obtained by moving with large step lengths, where the step lengths in each of the $M$ directions are controlled by a parameter $T$ called temperature. The step length and temperature are coupled so that as $T$ falls, step-size decreases. As the algorithm progresses and $T$ falls, it focuses on the most promising area within the parameter space. SA attempts to optimize the function while moving both uphill and downhill in order to escape local minima. Decisions on uphill movement also contain a random element. We use the implementation of SA for continuous variables described in [11].

Let us denote the vector containing the parameters to be optimized by $\bar{X}=\left[X_{1}, X_{2}, \ldots, X_{M}\right]^{T}$ and the cost function being minimized by $O(\bar{X})$. To optimize a cost function with SA, we first generate an initial guess $\bar{X}_{0}$ and calculate $O\left(\bar{X}_{0}\right)$. SA then generates a new guess $\bar{X}_{1}$ based on $\bar{X}_{0}, O\left(\bar{X}_{0}\right)$, and rules built into the SA algorithm. This process in which new parameters are generated based on past parameters is repeated a preset number of times after which the optimal parameter value $\bar{X}^{O P T}$ is reported. This optimal value is chosen so that $O\left(\bar{X}^{O P T}\right) \leq O\left(\bar{X}_{m}\right)$ for any $m$.

In the context of optimizing circuit parameters which minimize memory access time and energy usage, we let $O(\bar{X})$ be access time or energy as defined in the following section. The parameter vector $\bar{X}$ is composed of parameters such as external DC pulse value or external AC pulse amplitude and width. Notice that the value of each cost function depends on the solution to Equation (1), which in turn depends on the values of $\bar{X}$. As such, we couple the SA algorithm with a numerical solution of Equation (1) computed using an adaptive Runge-Kutta scheme.

One of the criteria for the parameter optimization process is to choose parameter sets in such a way that the pulse is similar in amplitude to a single flux quantum (SFQ) pulse generated by the pulse trigger, avoiding the need for SFQ pulse amplification. The SA algorithm allows one to include such constraints. Moreover, when optimization parameters do not yield solutions to Equation (1) which are appropriate (e.g., the solution does not result in the correct memory operation) we penalize the cost function and define $O(\bar{X})$ to be 2-3 orders of magnitude larger than a typical value of $O(\bar{X})$.

There are a number of reasons why we use SA rather than other optimization schemes. At a fixed temperature, SA performs random walks through the parameter search space, accepting both uphill and downhill moves with a predetermined probability. By accepting both uphill and downhill moves, SA avoids many local minima, though at an increased computational cost. Along with avoiding local minima, SA has other advantages which suggest its use for the optimization of Josephson junction parameters. 
First, unlike many iterative optimization schemes (e.g., Newton or steepest-descent schemes), SA can produce accurate results even for bad choices of initial conditions. Moreover, SA makes no assumption that a cost function is continuous. This property is important because our cost functions are discontinuous as explained below. Finally, SA can be used on cost functions of arbitrary numbers of variables. As we will see, SA effectively approximates global minima for the cost functions introduced in the previous section. For more details about SA and its applications, see, for example, [11-13].

\section{Definition of cost functions}

We are interested in system parameters which minimize both access time and energy usage for Read, Write, and Reset operations in the Josephson junction memory circuit. Here we explicitly define how these are calculated.

Access time: a Write operation has taken place when the voltage of a junction in response to external pulse exceeds some pre-defined value and is near its maximum value. As such, access time for a particular junction is defined as the difference between the time at which the voltage of that junction is maximized, denoted by $\tau_{j, \max }$, and the time at which the pulse is first non-zero, denoted by $\tau_{j, 0}$. Denote the width of the AC pulse which is applied to the $j^{\text {th }}$ junction by $\sigma_{j}$. Because a Gaussian pulse is always non-zero, we approximate the time at which the pulse is first non-zero by $\tau_{j, 0}=-\sigma_{j} / 2$. Access time, $T_{j}$, is defined by $T_{j}=\tau_{j, \max }-\tau_{j, 0}-\tau_{j}=\tau_{j, \max }-\tau_{j}+\sigma_{j} / 2$ where we recall that $\tau_{j}$ is the time-lag parameter in the AC pulse.

Energy dissipation: within the RSJ model for Josephson junctions, the expression for energy dissipation in the circuit can be written as [14]

$$
E^{\text {circuit }}=\sum_{j=1}^{3} \int_{0}^{\infty} I_{R_{j}} V_{j} d \tau=\sum_{j=1}^{3} \int_{0}^{\infty} V_{j}^{2} / R_{j} d \tau=\sum_{j=1}^{3} \int_{0}^{\infty}\left(\frac{\hbar}{2 e}\right)^{2} \frac{1}{R_{j}}\left(\frac{d \phi_{j}}{d \tau}\right)^{2} d \tau
$$

Energy dissipation only occurs between the time of pulse arrival (which we take as $\tau=0$ ) and when the system relaxes back to zero-voltage state. Here $I_{R_{j}}$ denotes resistive current (both external DC current and pulse current).

A different way to approximate energy dissipation [14] is to assume that at the time of pulse operation the highest portion of the external current will be flowing on the resistor due to non-zero voltage on each junction in the array. Thus,

$$
\begin{aligned}
\text { Eresistor }=\sum_{j=1}^{3} \int_{0}^{\infty} I_{R_{j}} V_{j} d \tau & \\
& \approx \sum_{j=1}^{3} I_{\text {total }} \int_{0}^{\infty} V_{j} d \tau \\
& =I_{\text {total }} \int_{0}^{\infty} \frac{\hbar}{2 e} \frac{d \phi_{j}}{d \tau} d \tau \approx I_{D C} \sum_{j=1}^{3} \int_{0}^{\infty} \frac{\hbar}{2 e} d \phi_{j}=\frac{\hbar I_{D C}}{2 e} \sum_{j=1}^{3}\left(\phi_{j, \text { final }}-\phi_{j, \text { initial }}\right)
\end{aligned}
$$

In dimensionless units this is

$$
E^{\text {resistor }}=\sum_{j=1}^{3} \int_{0}^{\infty} \gamma_{j} V_{j}^{2}(\tau) d \tau \text {. }
$$

Later, we will refer to (2) as total circuit energy and (3) as total resistor energy (or sometimes just total energy). Typically, we will report values for total resistor energy. 
When computing these values in practice, we integrate only until each junction's voltage is within a predefined tolerance of zero. Moreover, in the above equations the pulse is assumed to be zero until time $\tau=0$.

Pulse energy: pulse energy is the amount of energy used by the Josephson array dissipated over the amount of time required to access junction $j$. Again denote current in the $k^{\text {th }}$ junction by $i_{k}(\tau)$ and voltage in the $k^{\text {th }}$ junction by $V_{k}(\tau)$. Pulse energy for junction $j, E_{j}$, is defined by [14]

$$
E_{j}=\sum_{k=1}^{3} \int_{0}^{T_{j}} \gamma_{k} V_{k}^{2}(\tau) d \tau
$$

As before, $T_{j}$ is the junction access time for the $j^{\text {th }}$ junction. Again, we assume that the pulse is zero until time $\tau=0$.

Since only the third junction moves when a Write ' 0 ' operation is applied, access for Write ' 0 ' operations is only calculated for the third junction. For similar reasons, Write ' 1 ' access times are calculated for both the first and second junctions. Note that in all cases, we optimize Write ' 0 ' pulses only for the operation of moving the Josephson junction from phase $\{1,1,0\}$ to phase $\{1,1,1\}$. For Write ' 1 ' operations, we only optimize cost functions for the operation of moving the Josephson junction from phase $\{0,0,0\}$ to phase $\{1,1,0\}$.

\section{Overview of results}

The primary focus of this research was to find parameter values that lead to reduced access times and energies. Three sets of parameters were studied with this goal in mind: first, we investigated the Gaussian pulse parameters of amplitude and width for Write ' 0 ' and Write ' 1 ' operations holding all other parameters fixed. By focusing on a small parameter range, we were able to visually assess that the simulated annealing algorithm produced acceptable results. Second, we studied the effect the coupling constant $\kappa$ has on reducing cost functions as well as the amplitude of Gaussian pulses. Finally, we optimized both pulse parameters and DC parameters to further reduce access times and energies. DC parameters were constrained to ensure that phase $\{0,0,0\}$ state was still acceptable as a ' 0 ' state and state $\{1,1,0\}$ was still acceptable as a ' 1 ' state. Each of these will be discussed extensively below. First, however, we start with an overview of the optimization results.

Note that in the overview, we only give optimal values of the cost functions, rather than the circuit parameters which lead to these values. The reason for this temporary omission is related to circuit robustness with respect to fabrication imperfections, as discussed in the Introduction. In particular, since we do not have precise knowledge of errors introduced in the manufacturing process, we do not wish to recommend precise circuit parameter values to be used in circuit fabrication. Nonetheless, our numerical simulations suggest that a large range of circuit parameter values results at about the same values of the cost function. In the "Optimization Results" Section, we display heat maps which suggest this robustness. If better knowledge of the manufacturing process is obtained, that information can be built into the optimization procedure described previously.

\section{Gaussian pulse optimization:}

We optimized Gaussian pulse parameters over $0 \mu A \leq i_{A C, k} \leq 800 \mu A$ and $0.0415 p s \leq \sigma_{k} \leq 6.225 p s$ with $\kappa_{1}=\kappa_{2}=0.1, i_{D C, 1}=1.0, i_{D C, 2}=0.8$, and $i_{D C, 3}=-1.0$. The amplitudes allowed here are larger than those allowed in the experiments in later sections. While this leads to improved cost function values, it also may require a large adjustment of a pulse coming from the trigger. 
For Write ' 0 ' operations, optimal values for access times are near $2 \mathrm{ps}$, total energy optimal values are near $9 \times 10^{-19} \mathrm{~J}$, and optimal pulse energies are near $8 \times 10^{-19}$.

For Write ' 1 ' operations, optimal access times for junction one are near 30 ps. For junction two, they are approximately $95 \mathrm{ps}$. Minimum total energy is approximately $2 \times 10^{-18} \mathrm{~J}$. Optimal pulse energy for junction one is near $9 \times 10^{-19} \mathrm{~J}$ and for junction two near $1.7 \times 10^{-18}$.

\section{Constrained size Gaussian pulse optimization:}

The following results come from a parameter range of $0 \mu A \leq i_{A C, k} \leq 300 \mu A$ and $0.0415 p s \leq \sigma_{k} \leq$ 1.0375 ps.

- $\quad \kappa=0.05, i_{D C, 1}=i_{D C, 2}=1.0, i_{D C, 3}=0.7$ :

Minimum access time for Write ' 0 ' operations is $15 \mathrm{ps,} \mathrm{minimum} \mathrm{total} \mathrm{energy} \mathrm{for} \mathrm{Write} \mathrm{'} 0$ ' operation is approximately $1.1 \times 10^{-18} \mathrm{~J}$, and minimum pulse energy for Write ' 0 ' operations is near $6.0 \times 10^{-19} \mathrm{~J}$.

Minimum access time for a Write ' 1 ' operation evaluated on junction one is approximately 36 ps. For junction two, minimum access time is approximately $65 \mathrm{ps}$. Total energy for a Write ' 1 ' operation has a minimum value near $2.4 \times 10^{-18} \mathrm{~J}$, pulse energy on junction one has a minimum value of $6 \times 10^{-19} \mathrm{~J}$, and on junction two the minimum pulse energy is $1.8 \times 10^{-18} \mathrm{~J}$.

- $\quad \kappa=0.15, i_{D C, 1}=i_{D C, 2}=1.0, i_{D C, 3}=0.2$ :

Minimum access time for Write ' 0 ' operations is $14 \mathrm{ps,} \mathrm{minimum} \mathrm{total} \mathrm{energy} \mathrm{for} \mathrm{Write} \mathrm{'} 0$ ' operations is approximately $8.9 \times 10^{-19} \mathrm{~J}$, and minimum pulse energy for Write ' 0 ' operations is near $4.7 \times 10^{-19} \mathrm{~J}$.

Minimum access time for a Write ' 1 ' operation evaluated on junction one is approximately 22 ps. For junction two, minimum access time is approximately $41 \mathrm{ps}$. Total energy for a Write ' 1 ' operation has a minimum value near $2.0 \times 10^{-18}$, pulse energy on junction 1 has a minimum value of $5 \times 10^{-19} \mathrm{~J}$, and on junction two the minimum pulse energy is $1.6 \times 10^{-18} \mathrm{~J}$.

- $\quad \kappa=0.25, i_{D C, 1}=i_{D C, 2}=1.0, i_{D C, 3}=-0.18$ :

Minimum access time for Write ' 0 ' operations is $13 \mathrm{ps,} \mathrm{minimum} \mathrm{total} \mathrm{energy} \mathrm{for} \mathrm{Write} \mathrm{'} 0$ ' operations is approximately $7.2 \times 10^{-19} \mathrm{~J}$, and minimum pulse energy for Write ' 0 ' operations is near $4 \times 10^{-19} \mathrm{~J}$.

Minimum access time for a Write ' 1 ' operation evaluated on junction one is approximately 21 ps. For junction two, minimum access time is approximately $38 \mathrm{ps}$. Total energy for a Write ' 1 ' operation has a minimum value near $1.8 \times 10^{-18} \mathrm{~J}$, pulse energy on junction 1 has a minimum value of $4.381 \times 10^{-19} \mathrm{~J}$, and on junction two the minimum pulse energy is $1.30 \times 10^{-18} \mathrm{~J}$.

\section{Full current optimization:}

For the following optimization procedures we used the values of $\kappa=0.1$. Because we optimize both AC and DC parameters, we define a valid set of DC parameters as one which allows for both Write ' 0 ' and Write ' 1 ' operations to take place. This also requires us to change cost functions: we use a sum of the cost function for a Write ' 0 ' operation with the cost function for a Write ' 1 ' operation. We compute this sum with Write ' 1 ' cost functions evaluated at both the first and second junction. We run the optimization routines with the constraints $90 \mu \mathrm{A} \leq i_{D C, 1} \leq 100 \mu \mathrm{A}, 75 \mu \mathrm{A} \leq i_{D C, 2} \leq 100 \mu \mathrm{A},-100 \mu \mathrm{A} \leq i_{D C, 3} \leq$ $40 \mu \mathrm{A}, 0 \mu \mathrm{A} \leq i_{A C, k} \leq 300 \mu \mathrm{A}$, and $0.0415 p s \leq \sigma_{k} \leq 1.0375$ ps.

Access time for a Write ' 0 ' operation when optimizing a Write ' 1 ' operation on the first junction is approximately $15 \mathrm{ps}$. When optimizing the Write ' 1 ' operation on the second junction, optimal access time is also approximately $15 \mathrm{ps}$. Total energy for a Write ' 0 ' operation is minimized near $8.8 \times 10^{-19} \mathrm{~J}$ 
and pulse energy when optimizing a Write ' 1 ' operation on the first junction is approximately $5.1 \times$ $10^{-19} \mathrm{~J}$. When optimizing a Write ' 1 ' operation on the second junction, pulse energy for a Write ' 0 ' operation is near $5.4 \times 10^{-19} \mathrm{~J}$.

For a Write ' 1 ' operation, access time when optimizing on the first junction is approximately $27 \mathrm{ps.} \mathrm{When}$ optimizing on the second junction, minimum access time is approximately $50 \mathrm{ps}$. The value of the minimum total energy is approximately $1.9 \times 10^{-18} \mathrm{~J}$. When optimized on the first junction, minimum pulse energy is approximately $5.4 \times 10^{-19} \mathrm{~J}$ and when optimized on the second junction, minimum pulse energy is near $1.5 \times 10^{-18} \mathrm{~J}$.

Before continuing with more detailed optimization results we give some analytic justification of our results.

\section{Analytical Verification}

\section{Total energy}

By approximating the shape of the solution to the differential equation governing the Josephson junction, $\phi_{j}(\tau)$, we are able to obtain an order-of-magnitude approximation to total dissipation energy, which we calculate here using both definitions of total energy. In particular, we estimate each $\phi_{j}(\tau)$ by a piecewise linear function which moves from the initial equilibrium position of $\phi_{j}(\tau)$ to the resting equilibrium position of $\phi_{j}(\tau)$. We assume that this motion occurs in the calculated access time for each junction and that each $\phi_{j}(\tau)$ is zero outside of this time. If we denote the equilibrium position of the $j^{\text {th }}$ phase at state ' 0 ' by $\phi_{j}^{(e q, 0)}$, the equilibrium position of the $j^{\text {th }}$ phase at state ' 1 ' by $\phi_{j}^{(e q, 1)}$, and the access time of the $j^{\text {th }}$ junction by $T_{j}$, then

$$
\begin{aligned}
& \phi_{1}(\tau) \approx\left\{\begin{array}{c}
\phi_{1}^{(e q, 0)}, \tau \leq 0, \\
\phi_{1}^{(e q, 0)}+\frac{1}{T_{1}}\left(\phi_{1}^{(e q, 1)}-\phi_{1}^{(e q, 0)}\right) \tau, \quad 0 \leq \tau \leq T_{1}, \\
\phi_{1}^{(e q, 1)}, \quad T_{1} \leq \tau,
\end{array}\right. \\
& \phi_{2}(\tau) \approx\left\{\begin{array}{c}
\phi_{2}^{(e q, 0)}, \quad \tau \leq T_{2}, \\
\frac{1}{T_{2}-T_{1}}\left(\phi_{2}^{(e q, 1)}\left(\tau-T_{1}\right)+\phi_{2}^{(e q, 0)}\left(T_{2}-\tau\right)\right), \quad T_{1} \leq \tau \leq T_{2}, \\
\varphi_{2}^{(e q, 1)}, T_{2} \leq \tau, \text { and }
\end{array}\right.
\end{aligned}
$$

$\phi_{3}(\tau) \approx\left\{\begin{array}{c}\phi_{3}^{(e q, 0)}, \quad \tau \leq T_{2}, \\ \frac{1}{T_{3}-T_{2}}\left(\phi_{3}^{(e q, 1)}\left(\tau-T_{2}\right)+\phi_{3}^{(e q, 0)}\left(T_{3}-\tau\right)\right), \quad T_{2} \leq \tau \leq T_{3}, \\ \phi_{3}^{(e q, 1)}, \quad T_{3} \leq \tau .\end{array}\right.$

To estimate access energy, we do not include the contribution of the AC pulse to energy. While the AC pulse certainly contributes to energy, this contribution takes place over a short time, so we ignore it and note that our calculation will underestimate the true value of energy. With these approximations in hand, using the definition of energy which we denoted by total circuit energy, we have 


$$
\begin{aligned}
& E^{\text {circuit }}=\int_{0}^{T} \sum_{k=1}^{3} i_{k}(\tau) \phi_{k}^{\prime}(\tau) d \tau \\
& \approx \int_{0}^{T_{1}} \frac{i_{D C, 1}}{T_{1}}\left(\phi_{1}^{(e q, 1)}-\phi_{1}^{(e q, 0)}\right) d \tau+\int_{T_{1}}^{T_{2}} \frac{i_{D C, 2}}{T_{2}-T_{1}}\left(\phi_{2}^{(e q, 1)}-\phi_{2}^{(e q, 0)}\right) d \tau \\
&+\int_{T_{2}}^{T_{3}} \frac{i_{D C, 3}}{T_{3}-T_{2}}\left(\phi_{3}^{(e q, 1)}-\phi_{3}^{(e q, 0)}\right) d \tau \\
&=i_{D C, 1}\left(\phi_{1}^{(e q, 1)}-\phi_{1}^{(e q, 0)}\right)+i_{D C, 2}\left(\phi_{2}^{(e q, 1)}-\phi_{2}^{(e q, 0)}\right) \\
&+i_{D C, 3}\left(\phi_{3}^{(e q, 1)}-\phi_{3}^{(e q, 0)}\right) .
\end{aligned}
$$

Using $i_{D C, 1}=1.0, i_{D C, 2}=0.8$, and $i_{D C, 3}=-1.0$ and approximating equilibrium positions for each junction as calculated in [9], we calculate

$$
E^{\text {circuit }} \approx 9.868=1.9736 \times 10^{-18} \mathrm{~J} .
$$

Compared to the value calculated numerically for a sample Write ' 1 ' pulse with $i_{\text {pulse. } 1}=1.0, \sigma_{1}=1.0$, $E^{\text {circuit }}=10.572=2.114 \times 10^{-18} \mathrm{~J}$, we see good agreement despite the somewhat large approximations used in the approximation. Further numerical experiments suggest that the method described above typically produces values with approximately $30 \%$ error compared to simulated values.

In exactly the same way, we can approximate total resistor energy. In particular,

$$
\begin{aligned}
& E^{\text {resistor }}=\int_{0}^{\infty} \sum_{k=1}^{3} \gamma_{k}\left(\phi_{k}^{\prime}(\tau)\right)^{2} d \tau \approx \int_{0}^{T_{1}} \frac{\gamma_{1}}{T_{1}^{2}}\left(\phi_{1}^{(e q, 1)}-\phi_{1}^{(e q, 0)}\right)^{2} d \tau+\int_{T_{1}}^{T_{2}} \frac{\gamma_{2}}{\left(T_{2}-T_{1}\right)^{2}}\left(\phi_{2}^{(e q, 1)}-\right. \\
& \left.\phi_{2}^{(e q, 0)}\right)^{2} d \tau+\int_{T_{2}}^{T_{3}} \frac{\gamma_{3}}{\left(T_{3}-T_{2}\right)^{2}}\left(\phi_{3}^{(e q, 1)}-\phi_{3}^{(e q, 0)}\right)^{2} d \tau=\frac{\gamma_{1}}{T_{1}}\left(\phi_{1}^{(e q, 1)}-\phi_{1}^{(e q, 0)}\right)^{2}+\frac{\gamma_{2}}{T_{2}-T_{1}}\left(\phi_{2}^{(e q, 1)}-\right. \\
& \left.\phi_{2}^{(e q, 0)}\right)^{2}+\frac{\gamma_{3}}{T_{3}-T_{2}}\left(\phi_{3}^{(e q, 1)}-\phi_{3}^{(e q, 0)}\right)^{2} .
\end{aligned}
$$

Approximating equilibrium positions for each junction using equilibrium fixed points as above and using the approximations of $T_{j}$ also calculated in [9], we approximate total energy. For $i_{\text {pulse.1 }}=1.0, \sigma_{1}=1.0$ and all other parameters the same as in previous calculations, we find

$$
E^{\text {resistor }} \approx 7.41=1.54 \times 10^{-18} \mathrm{~J} \text {. }
$$

Compared to the numerically calculated value for a sample Write ' 1 ' pulse with, $E^{\text {resistor }}=9.108=$ $1.90 \times 10^{-18} \mathrm{~J}$, we see good agreement despite the somewhat large approximations used in the calculations. As before, we see around 30\% error compared with numerical results.

\section{Optimization results}

We now report the results of various optimization schemes in detail. Along with numerical results from the SA algorithm, we show images which indicate that the results are relatively robust to noise.

\section{Gaussian pulse parameter optimization}

We begin with optimization of external pulse values for Write ' 0 ' and Write ' 1 'operations. We first optimize these values over a large range of possible values, both to demonstrate the feasibility of the optimization scheme and to suggest a lower limit on expected values for access times and energies. Next, 
we constrain the values which pulse amplitudes and widths can take to more realistic levels. We also change $\kappa_{1}$ and $\kappa_{2}$ to show that access times and energies are relatively robust to this value.

We list estimates to the optimal values of pulse amplitudes and widths obtained with the SA algorithm to minimize access times and energies. We also show a coarse plot of both access times and energies as a function of these pulse parameters. All figures are heat maps of the cost functions being optimized as a function of pulse amplitude and width. For clarity, pulse amplitude in the figures is defined as the maximum value of the applied pulse, not the value $i_{p u l s e, j}$ (i.e., the value given in figures is $i_{p u l s e, j} /$ $\sqrt{2 \pi \sigma_{j}^{2}}$ ). Note that in all figures, locations on the heat map which are white correspond to pulse parameter values which do not behave as a the type of operation we are simulating, e.g., for a Write ' 0 ' simulation, the pulse does not move a junction from state $\{1,1,0\}$ to state $\{1,1,1\}$.

Initially, we consider $\kappa_{1}=\kappa_{2}=0.1$ and set $i_{D C, 1}=1.0, i_{D C, 2}=0.8, i_{D C, 3}=-1.0$.

Write 'O' operation

The plot in Figure 2 is a heat map of Write ' 0 ' access times, total energies, and pulse energies for junction three as a function of pulse width and pulse amplitude. Access times are in the range of $1-12 \mathrm{ps}$. These times are very small due to both the large amplitude of Gaussian pulse and because only one junction needs to move for a Write ' 0 ' operation to take place. Total energies for Write ' 0 ' operations are typically between $8 \times 10^{-19} \mathrm{~J}$ and $6 \times 10^{-18} \mathrm{~J}$ while pulse energies for Write ' 0 ' operations are in the range $1 \times$ $10^{-19}-2 \times 10^{-18} \mathrm{~J}$. The optimal access time of around 2 ps occurs at pulse amplitude near $800 \mu \mathrm{A}$ and width near 1.1 ps. An optimal total energy is near $9 \times 10^{-19} \mathrm{~J}$ and occurs with pulse amplitude near $346 \mu \mathrm{A}$ and width near $3.15 \mathrm{ps}$. Finally, optimal pulse energy is approximately $8 \times 10^{-19} \mathrm{~J}$ resulting from pulse amplitude of approximately $316 \mu \mathrm{A}$ and width of $3.7 \mathrm{ps}$.
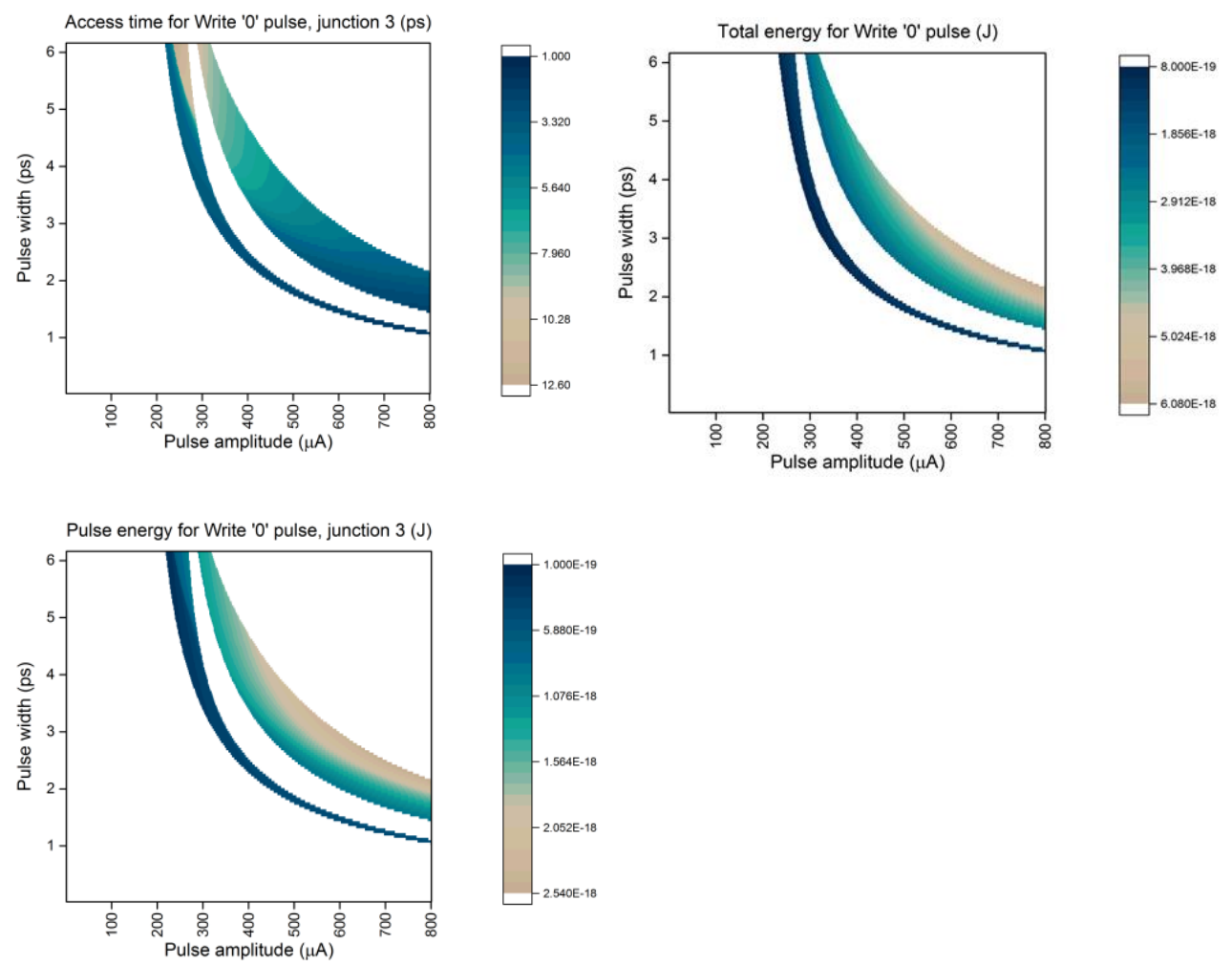

Figure 2: Heat maps of cost functions for Write ' 0 ' operations. (Top Left) Access time for junction 3. (Top Right) Total energy for junction 3. (Bottom) Pulse energy for Write '0' pulse. 


\section{Write '1' operation}

Figure 3 is a heat map of the access times and total energies for Write ' 1 ' operations. Typical access times for junction one are in the range of $29-55 \mathrm{ps}$ and for junction two in the range $90-120 \mathrm{ps}$. Access times for junction two are so much larger than for junction one because two junctions must move for junction two to be accessed, while only one needs to move for junction one to be accessed. Total energies are all near $2 \times 10^{-18} \mathrm{~J}$. Optimal access times for junction one are near $30 \mathrm{ps}$ and occur for pulse amplitudes of approximately $100 \mu A$ and widths near 1.7 ps. For junction two, optimal access times are approximately 95 ps with amplitudes near $58 \mu A$ and widths near 3.07 ps. Minimum total energy is approximately $2 \times 10^{-18} \mathrm{~J}$ and this occurs for pulse amplitudes near $320 \mu A$ and widths near $0.33 \mathrm{ps}$.
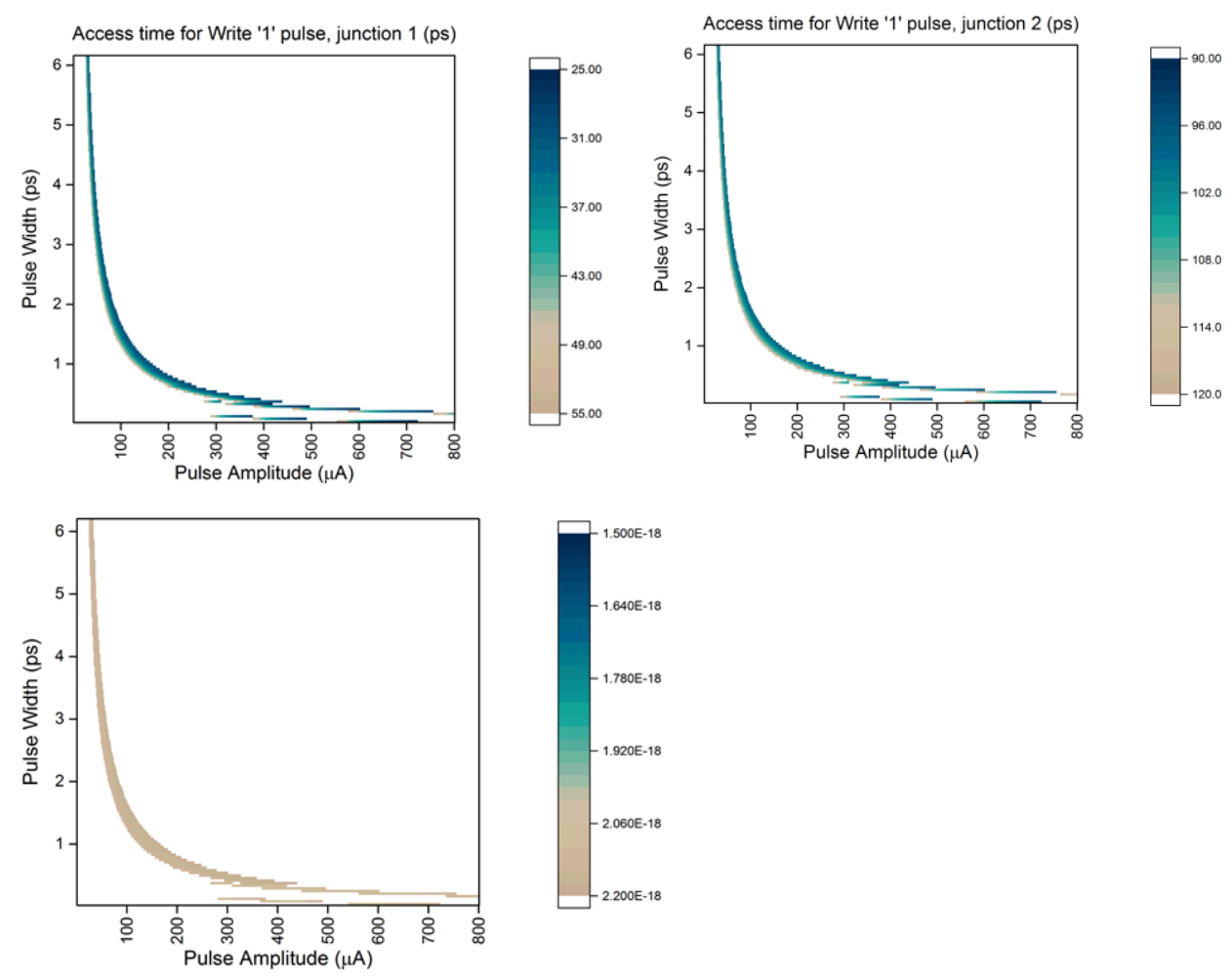

Figure 3: Heat maps of cost functions for Write ' 1 ' operations. (Top Left) Access time for junction 1. (Top Right) Access time for junction 2. (Bottom) Total energy for Write ' 1 ' pulse.

The heat maps in Figure 4 show the pulse energies for junction one and junction two. Pulse energies for junction one are all near $1 \times 10^{-18} \mathrm{~J}$ and for junction two near $2 \times 10^{-18} \mathrm{~J}$. For junction one, pulse amplitudes near $325 \mu \mathrm{A}$ and widths near 0.33 ps give minimum pulse energies near $9 \times 10^{-19} \mathrm{~J}$ while for junction two, minimum pulse energies of approximately $1.7 \times 10^{-18} \mathrm{~J}$ are produced by pulses with amplitudes of approximately $335 \mu \mathrm{A}$ and widths of approximately $0.32 \mathrm{ps}$. 

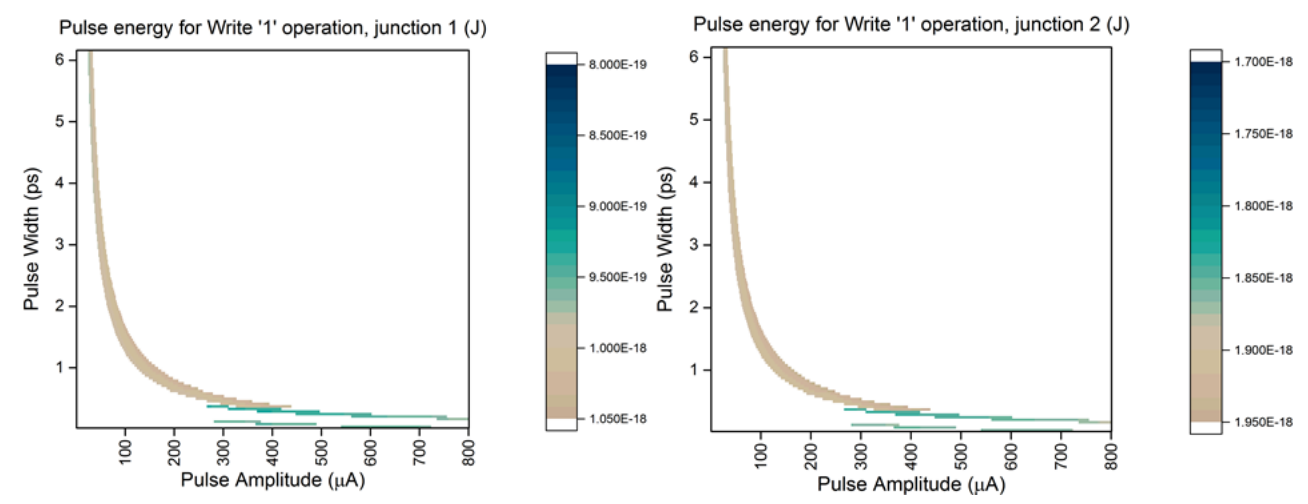

Figure 4: Heat map of pulse energies for a Write ' 1 ' operation. Pulse energy for junction 1 (right) and for junction 2 (left).

\section{Effect of the pulse amplitude constraints on optimization}

In some occasions, it may be important to restrict pulse amplitude and width to certain values (for example to avoiding the need for pulse amplification). In this section we present optimization results for pulse amplitudes restricted to be smaller than $300 \mu \mathrm{A}$ and pulse widths to be smaller than $1.0375 \mathrm{ps}$. The coupling parameter $\kappa$ and the DC value $i_{D C, 3}$ are adjusted to allow for this constraint. We fix $i_{D C, 1}=$ $i_{D C, 2}=1.0$. We also vary $\kappa$ and $i_{D C, 3}$ to show that values for access times and energies are relatively stable for different choices of these parameters. We only report cost function results for Write ' 1 ' operations at junction two, as these are more useful in practice.

In Figure 5, we present heat maps of cost function values as a function of Gaussian pulse width and amplitude. These values were calculated with $\kappa=0.05, i_{D C, 1}=i_{D C, 2}=1.0$, and $i_{D C, 3}=0.7$. Typical access times for Write ' 0 ' pulses are in the range of 10-80 ps while typical access times for Write ' 1 ' pulses are in the range of 60-120 ps. Total energies for Write ' 0 ' pulses are in the range of $9 \times 10^{-19}-$ $1.3 \times 10^{-18} \mathrm{~J}$ while for Write ' 1 ' pulses, total energies fall in the range $2 \times 10^{-18}-2.5 \times 10^{-18} \mathrm{~J}$. Finally, pulse energies for junction three following a Write ' 0 ' pulse are near $4 \times 10^{-19}-8 \times 10^{-19} \mathrm{~J}$ and for junction two following a Write ' 1 ' pulse are near $3.7 \times 10^{-18} \mathrm{~J}$.

The optimal value for Write ' 0 ' access times is approximately $15 \mathrm{ps,} \mathrm{which} \mathrm{occurs} \mathrm{for} \mathrm{pulse} \mathrm{amplitude} \mathrm{of}$ approximately $252 \mu \mathrm{A}$ and width of approximately $1 \mathrm{ps}$. The optimal access times for Write ' 1 ' operations on junction two are near 65 ps and this occurs with pulse amplitude of near $200 \mu A$ and width of approximately $0.37 \mathrm{ps}$. Minimum total energy for Write ' 0 ' operations is approximately $1.1 \times 10^{-18} \mathrm{~J}$. This occurs with pulse amplitudes around $168 \mu \mathrm{A}$ and width around $0.17 \mathrm{ps}$. Total energy for a Write ' 1 ' operation has a minimum value near $2.4 \times 10^{-18} \mathrm{~J}$, which occurs with pulse amplitudes of $242 \mu A$ and widths of $0.12 \mathrm{ps}$. Finally, minimum pulse energy for Write ' 0 ' operations is near $6.0 \times 10^{-19} \mathrm{~J}$. This occurs with pulse amplitudes of $159 \mu \mathrm{A}$ and widths of $0.18 \mathrm{ps}$. For Write ' 1 ' operations on junction two, the minimum pulse energy is $1.9 \times 10^{-18} \mathrm{~J}$, which takes place for pulse amplitudes of $102 \mu A$ and widths of $0.19 \mathrm{ps}$. 

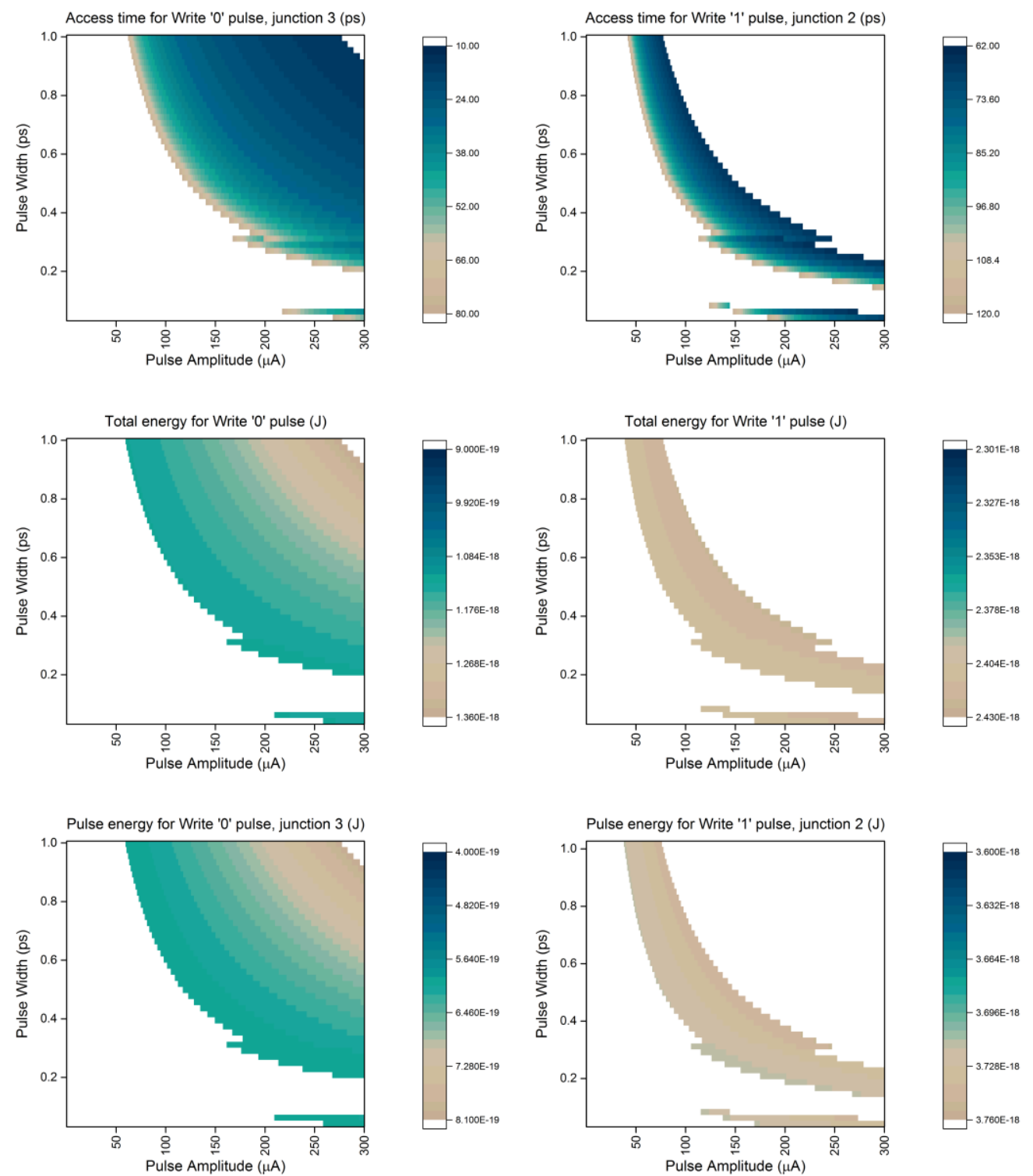

Figure 5: Heat maps of cost functions for $\kappa=0.05, i_{D C, 1}=i_{D C, 2}=1.0$, and $i_{D C, 3}=0.7$. (Top Row) Access times for Write ' 0 ' pulses and Write ' 1 ' pulses. (Middle Row) Total energies for Write ' 0 ' and Write ' 1 ' pulses. (Bottom Row) Pulse energies for Write ' 0 ' and Write ' 1 ' pulses.

In Figure 6, we show heat maps of the cost functions using $\kappa=0.15, i_{D C, 1}=i_{D C, 2}=1.0$, and $i_{D C, 3}=$ 0.2 . Typical access times for Write ' 0 ' pulses are in the range of 10-80 ps while typical access times for Write ' 1 ' pulses are in the range of 30-100 ps. Total energies for Write ' 0 ' pulses are in the range of $8 \times 10^{-19}-1 \times 10^{-18} \mathrm{~J}$ while for Write ' 1 ' pulses, total energies fall in the range $2 \times 10^{-18}-3 \times$ $10^{-18} \mathrm{~J}$. Finally, pulse energies for junction three following a Write ' 0 ' pulse are near $3 \times 10^{-19}-7 \times$ $10^{-19} \mathrm{~J}$ and for junction two following a Write ' 1 ' pulse are near $2.0 \times 10^{-18}-3.0 \times 10^{-18} \mathrm{~J}$. 

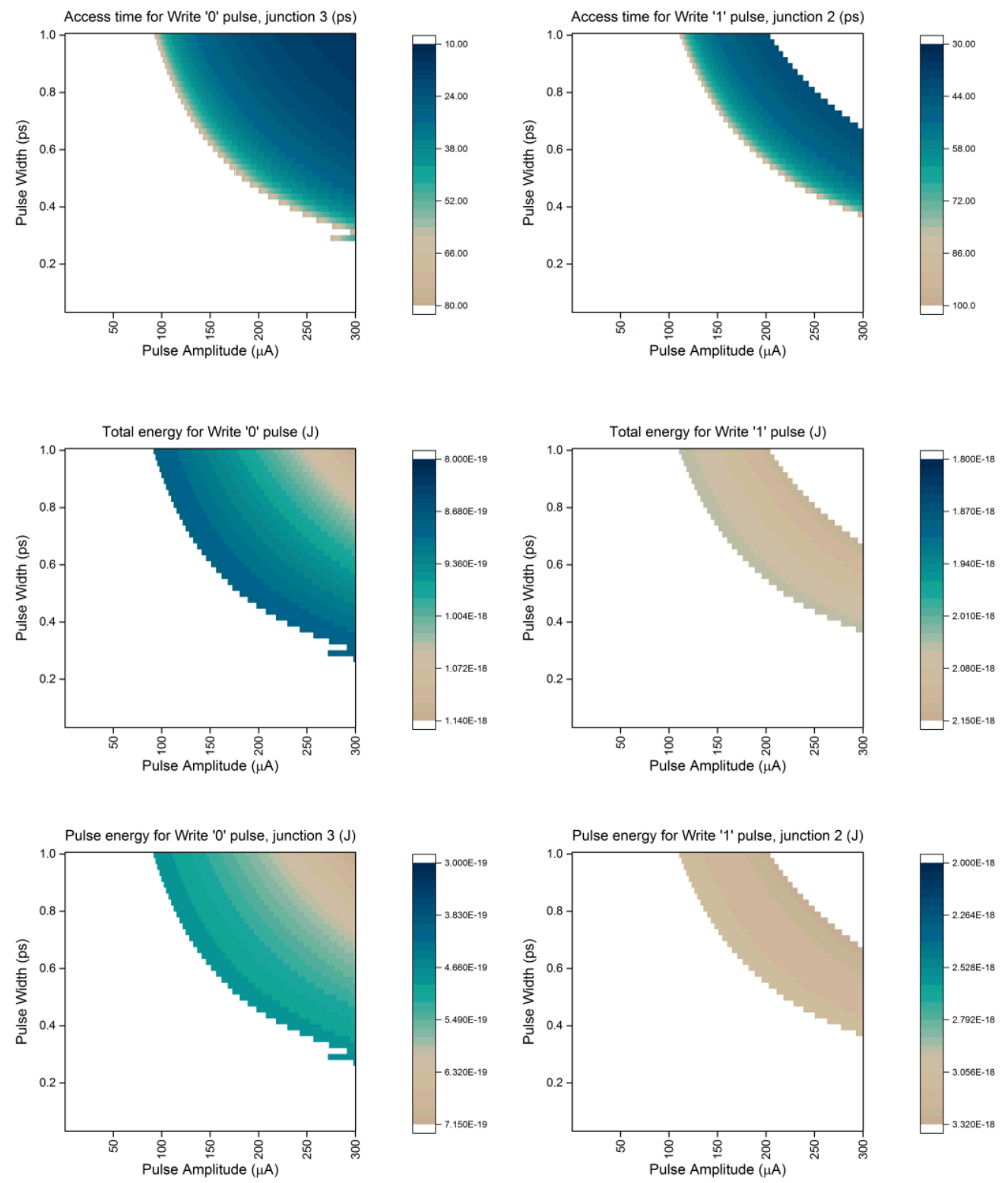

Figure 6: Heat maps of cost functions for $\kappa=0.15, i_{D C, 1}=i_{D C, 2}=1.0$, and $i_{D C, 3}=0.2$. (Top Row) Access times for Write ' 0 ' pulses and Write ' 1 ' pulses. (Middle Row) Total energies for Write ' 0 ' and Write ' 1 ' pulses. (Bottom Row) Pulse energies for Write ' 0 ' and Write ' 1 ' pulses.

The optimal value for Write ' 0 ' access times is approximately $14 \mathrm{ps,} \mathrm{which} \mathrm{occurs} \mathrm{for} \mathrm{pulse} \mathrm{amplitude} \mathrm{of}$ approximately $300 \mu \mathrm{A}$ and width of approximately $1 \mathrm{ps}$. The optimal access times for Write ' 1 ' operations on junction two are near $41 \mathrm{ps}$ and this occurs with pulse amplitude of near $233 \mu \mathrm{A}$ and width of approximately $0.88 \mathrm{ps}$. Minimum total energy for Write ' 0 ' operations is approximately $8.9 \times 10^{-19} \mathrm{~J}$. This occurs with pulse amplitudes around $88 \mu A$ and width around 1 ps. Total energy for a Write ' 1 ' operation has a minimum value near $2.0 \times 10^{-18} \mathrm{~J}$ which occurs with pulse amplitudes of $124 \mu A$ and widths of $0.89 \mathrm{ps}$. Finally, minimum pulse energy for Write ' 0 ' operations is near $4.7 \times 10^{-19} \mathrm{~J}$. This occurs with pulse amplitudes of $248 \mu A$ and widths of 0.18 ps. For Write ' 1 ' operations on junction two, the minimum pulse energy is $1.6 \times 10^{-18} \mathrm{~J}$, which takes place for pulse amplitudes of $107 \mu A$ and widths of $1.0 \mathrm{ps}$. 
In Figure 7, we show heat maps of cost function using $\kappa=0.25, i_{D C, 1}=i_{D C, 2}=1.0$, and $i_{D C, 3}=-0.18$. Typical access times for Write ' 0 ' pulses are in the range of 10-120 ps while typical access times for Write ' 1 ' pulses are in the range of 30-80 ps. Total energies for Write ' 0 ' pulses are in the range of $6 \times$ $10^{-19}-1 \times 10^{-18} \mathrm{~J}$ while for Write ' 1 ' pulses, total energies fall in the range $1 \times 10^{-18}-2 \times 10^{-18} \mathrm{~J}$. Finally, pulse energies for junction three following a Write ' 0 ' pulse are near $1 \times 10^{-19}-6 \times 10^{-19} \mathrm{~J}$ and for junction two following a Write ' 1 ' pulse are near $2 \times 10^{-18}-3 \times 10^{-18} \mathrm{~J}$.
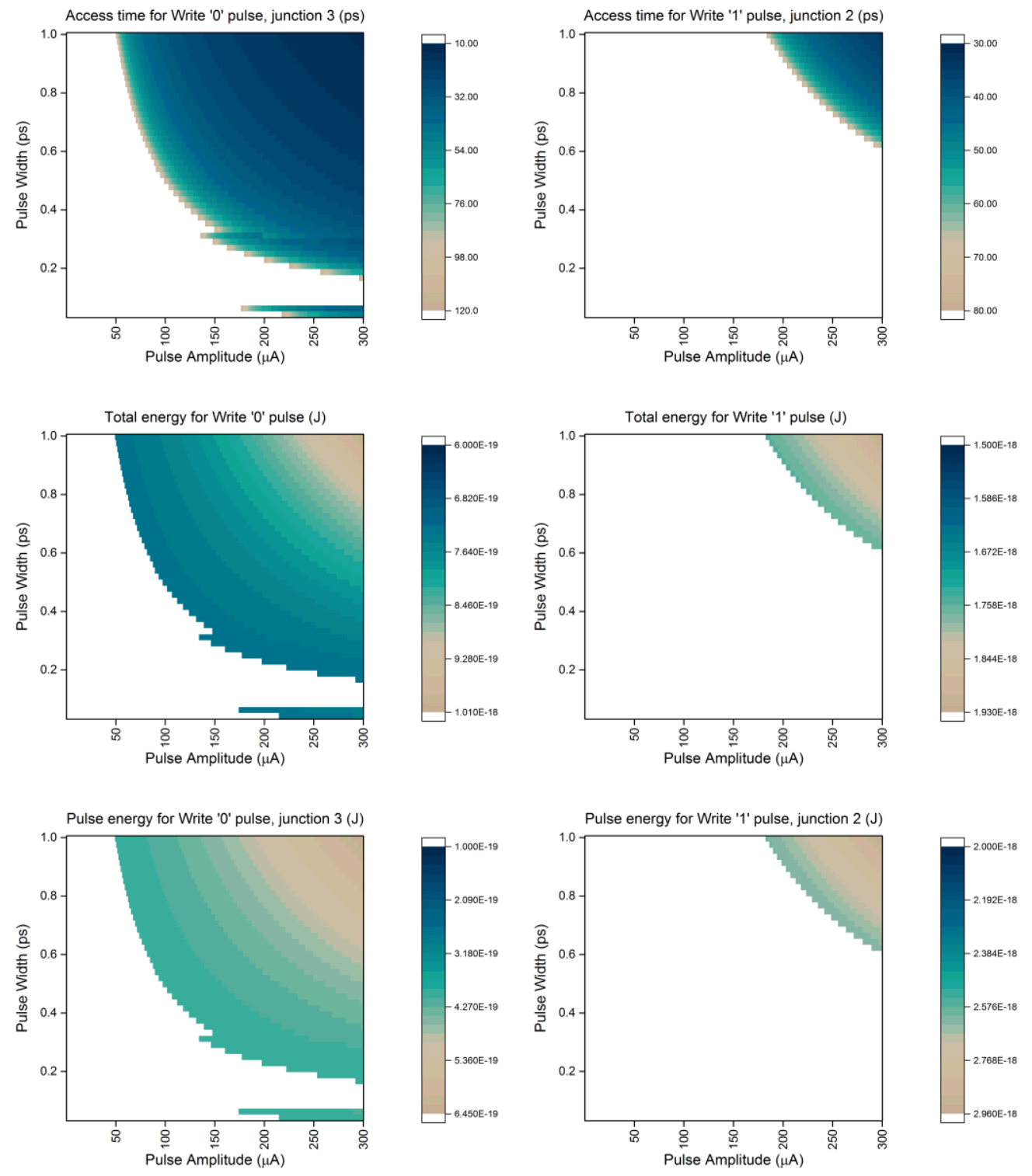

Figure 7: Heat maps of cost functions for $\kappa=0.25, i_{D C, 1}=i_{D C, 2}=1.0$, and $i_{D C, 3}=-0.18$. (Top Row) Access times for Write ' 0 ' pulses and Write ' 1 ' pulses. (Middle Row) Total energies for Write ' 0 ' and Write ' 1 ' pulses. (Bottom Row) Pulse energies for Write ' 0 ' and Write ' 1 ' pulses.

The optimal value for Write ' 0 ' access times is approximately $13 \mathrm{ps,} \mathrm{which} \mathrm{occurs} \mathrm{for} \mathrm{pulse} \mathrm{amplitude} \mathrm{of}$ approximately $300 \mu \mathrm{A}$ and width of approximately $1.0 \mathrm{ps}$. The optimal access times for Write ' 1 ' operations on junction two are near $38 \mathrm{ps}$ and this occurs with pulse amplitude of near $257 \mu \mathrm{A}$ and width of approximately $1.0 \mathrm{ps}$. Minimum total energy for Write ' 0 ' operations is approximately $7.2 \times 10^{-19} \mathrm{~J}$. This occurs with pulse amplitudes around $139 \mu A$ and width around $0.17 \mathrm{ps.} \mathrm{Total} \mathrm{energy} \mathrm{for} \mathrm{a} \mathrm{Write} \mathrm{'} 1$ ' 
operation has a minimum value near $1.8 \times 10^{-18} \mathrm{~J}$ which occurs with pulse amplitudes of $175 \mu A$ and widths of $1.0 \mathrm{ps}$. Finally, minimum pulse energy for Write ' 0 ' operations is near $4 \times 10^{-19} \mathrm{~J}$. This occurs with pulse amplitudes of $135 \mu \mathrm{A}$ and widths of $0.18 \mathrm{ps}$. For Write ' 1 ' operations on junction two, the minimum pulse energy is $1.3 \times 10^{-18} \mathrm{~J}$, which takes place for pulse amplitudes of $175 \mu \mathrm{A}$ and widths of 1.0 ps.

\section{DC current parameter optimization}

The final set of optimization numerical experiments (Figure 8) are to minimize access times and energies as functions of both AC pulse parameters and DC values. To do this, we first identify sets of DC parameters so that the proposed circuit can function in the same way as it does for fixed DC values. We require DC values so that the state $\{0,0,0\}$ can be thought of as a ' 0 ' state and $\{1,1,0\}$ can be thought of as a ' 1 ' state. Numerical experiments suggest that the constraints $90 \mu \mathrm{A} \leq i_{D C, 1} \leq 100 \mu \mathrm{A}, 75 \mu \mathrm{A} \leq$ $i_{D C, 2} \leq 100 \mu \mathrm{A}$, and $-100 \mu \mathrm{A} \leq i_{D C, 3} \leq 40 \mu \mathrm{A}$ are sufficient. The following four figures show regions in which different states exist as a function of $i_{D C, 1}$ for fixed values of $i_{D C, 2}$ and $i_{D C, 3}$. Notice that in all figures, when $90 \mu \mathrm{A} \leq i_{D C, 1} \leq 100 \mu \mathrm{A}$, there is a gap between state $\{0,0,0\}$ and state $\{1,1,0\}$ which has no other existing states. As such, the two states are able to act as ' 0 ' and ' 1 ' states, respectively.
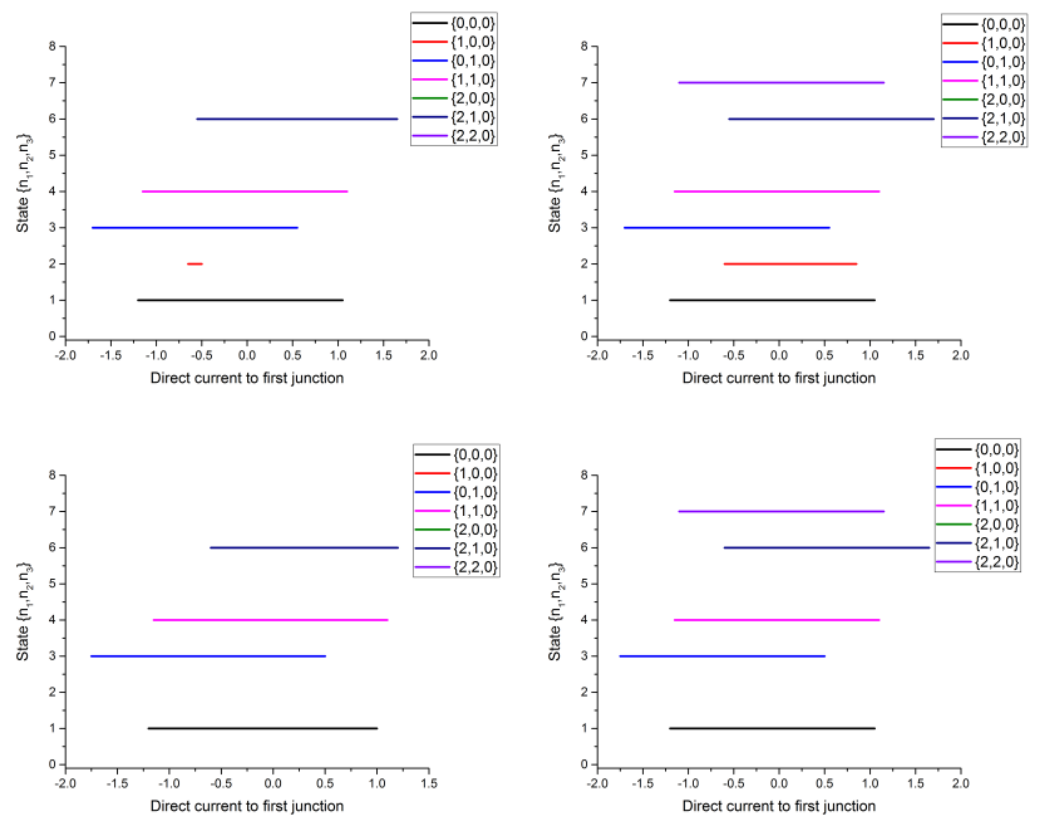

Figure 8: Stability diagrams as a function of DC to first junction for fixed DC values to second and third junction. Top left: $i_{D C, 2}=75 \mu \mathrm{A}, i_{D C, 3}=40 \mu \mathrm{A}$. Top right: $i_{D C, 2}=100 \mu \mathrm{A}, i_{D C, 3}=40 \mu \mathrm{A}$. Bottom left: $i_{D C, 2}=75 \mu \mathrm{A}, i_{D C, 3}=$ $-100 \mu \mathrm{A}$. Bottom right: $i_{D C, 2}=100 \mu \mathrm{A}, i_{D C, 3}=-100 \mu \mathrm{A}$.

One challenge in finding optimal DC parameters is that both Write ' 0 ' and Write ' 1 ' operations must exist and be viable for the chosen DC parameters. This condition forces a mild change in how cost functions are calculated. Each cost function is set to be very large for any combination of parameters such that either Write '0' or Write ' 1 ' operations are not valid. Moreover, for parameter values such that Write ' 0 ' and Write ' 1 ' operations are valid, cost functions are defined as the sum of the cost function for a Write ' 0 ' operation with the cost function for a Write ' 1 ' operation. For example, access time is defined as the sum of access time of a Write ' 0 ' operation on the third junction with the access time of a Write ' 1 ' operation on the first or second junction. 
Optimal cost functions for this set-up were calculated for a Write ' 0 ' operation evaluated on the third junction and for a Write ' 1 ' operation evaluated on the first junction or the second junction. In the first case, optimal access times occur for $i_{D C, 1}=97.1 \mu A, i_{D C, 2}=100 \mu A, i_{D C, 3}=40 \mu A$ and Write ' 0 ' pulse amplitudes near $300 \mu A$ and widths of 1 ps and Write ' 1 ' pulse amplitudes near $226 \mu A$ and width near 1 ps. Access time for a Write ' 0 ' operation with these parameters is approximately $15 \mathrm{ps}$ and for a Write ' 1 ' operation approximately $27 \mathrm{ps}$. Optimal access times evaluated from the third junction for Write ' 0 ' operations and from the second junction for Write ' 1 ' operations occur with $i_{D C, 1}=i_{D C, 2}=$ $100 \mu \mathrm{A}$, and $i_{D C, 3}=40 \mu \mathrm{A}$. Moreover, optimal Write ' 0 ' amplitudes are approximately $300 \mu \mathrm{A}$ and have widths of approximately $1 \mathrm{ps}$, while optimal Write ' 1 ' amplitudes are near $205 \mu A$ and widths are near 0.7 ps. The optimal Write ' 0 ' access time is around $15 \mathrm{ps}$ and the optimal Write ' 1 ' access time evaluated at the second junction is around $50 \mathrm{ps}$.

Total energy is minimized at $i_{D C, 1}=90.4 \mu A, i_{D C, 2}=75 \mu A$, and $i_{D C, 3}=15.7 \mu A$ with Write ' 0 ' pulse amplitudes of $288.85 \mu A$ and widths of 1.03 ps and Write ' 1 ' pulse amplitudes of $299 \mu A$ and widths of $0.62 \mathrm{ps}$. At these values, total energy for a Write ' 0 ' operation is near $8.83 \times 10^{-19} \mathrm{~J}$ and for a Write ' 1 ' operation approximately $1.9 \times 10^{-18} \mathrm{~J}$.

Finally, optimizing the sum of pulse energy from a Write ' 0 ' operation at the third junction and a Write ' 1 ' operation at the first junction takes its minimum value for parameters near $i_{D C, 1}=96.6 \mu A, i_{D C, 2}=$ $85.9 \mu A$, and $i_{D C, 3}=39.1 \mu A$. The optimal Write ' 0 ' pulse has amplitude near $299 \mu A$ and width near $0.37 \mathrm{ps}$ and the optimal Write ' 1 ' pulse has an amplitude near $127 \mu A$ and width near 1 ps. For this set-up, pulse energy for a Write ' 0 ' operation is near $5.14 \times 10^{-19} \mathrm{~J}$ and for a Write ' 1 ' operation it is approximately $5.36 \times 10^{-19} \mathrm{~J}$. Instead optimal values of the sum of a Write ' 0 ' operation at the third junction and a Write ' 1 ' operation at the second junction occur for $i_{D C, 1}=90.0 \mu A, i_{D C, 2}=75 \mu A$, and $i_{D C, 3}=29.5 \mu \mathrm{A}$. For this set-up, optimal Write ' 0 ' pulse amplitudes are approximately $221 \mu \mathrm{A}$ and widths are around 0.97 ps. Optimal Write ' 1 ' pulse amplitudes are near $216 \mu A$ and widths are near 0.9 ps. Pulse energy for a Write ' 0 ' operation is near $5.4 \times 10^{-19} \mathrm{~J}$ and for a Write ' 1 ' operation it is approximately $1.5 \times 10^{-18} \mathrm{~J}$.

\section{Summary and Discussion}

In this paper we presented results of an optimization process which used a simulated annealing algorithm to minimize memory access times and dissipation energies for a cryogenic memory circuit. We have employed Josephson junction parameter values that are consistent with the current state-of-the-art Josephson junction fabrication capabilities as presented in [10]. Our optimization process concentrated on studying the effect of external pulse amplitude and width, external DC currents, and array coupling constants on optimization and minimization of the circuit memory access times and access energies. We demonstrated stability and robustness of the memory circuit operation to fluctuations of junction internal parameters and external pulse and DC currents. For parameter values presented in the paper, access times are of the order of $30-100$ ps while dissipation energy is of the order of $5 \times 10^{-18}-10^{-19} \mathrm{~J}$.

Additional important issues must be addressed in order to better understand and design the proposed memory circuit. One issue is related to the feature size of the circuit that is related to the sizes of Josephson junctions and the value of the coupling constant $\kappa$ (which is inversely proportional to the value of the inductance). In our upcoming work we will study how to choose the values (and consequently the size) of the inductors in an optimal way to reduce the size of the circuit while simultaneously reducing memory access times and energies. The choice of Josephson junction parameters (and in particular $\gamma$ ) could also be implemented in an optimal way, which affects the circuit components, such as resistors and capacitors. Finally, the work presented in this manuscript was for a pre-fixed ' 0 ' and ' 1 ' state. In our future work, other choices for the states ' 0 ' and ' 1 ' should be explored in order to improve memory circuit operation and functionalities. 


\section{Acknowledgement}

This work was supported by the United States Department of Defense and used resources of the Computational Research and Development Programs of Oak Ridge National Laboratory. Oak Ridge National Laboratory is managed by UT-Battelle, LLC for the U.S. Department of Energy under Contract DE-AC05-00OR22725. We would like to acknowledge very valuable conversations and constructive feedback from Stephen Poole.

\section{References}

1. K. K. Likharev, Physica C 482, 6 (2012).

2. K. K. Likharev, Dynamics of Josephson junctions and circuits, Gordon and Breach science publishers, New York, (1986).

3. S. Nagasawa, K. Hinode, T. Satoh, Y. Kitagawa and M. Hidaka, Superconductor Science \& Technology 19, S325 (2006).

4. Q. Liu, K. Fujiwara, X. Meng, S. R. Whiteley, T. Van Duzer, N. Yoshikawa, Y. Thakahashi, T. Hikida, and N. Kawai, IEEE Transactions on Applied Superconductivity 17, 326 (2007).

5. O. A. Mukhanov, A. F. Kirichenko, T. V. Filippov, and S. Sarwana, IEEE Transactions of Applied Superconductivity 21, 797 (2011).

6. V. V. Ryazanov, V. V. Bol'ginov, D. S. Sobanin, I. V. Vernik, S. K. Tolpygo, A. M. Kadin and O. A. Mukhanov, Superconductivity Centennial Conference 2011 36, 35 (2012).

7. S. A. Holmes, L. Ripple and M. A. Manheimer (2013), IEEE Transactions on Applied Superconductivity 23, 1701610 (2013).

8. Y. Braiman, N. Nair, J. Rezac, and N. Imam, Superconductor Science and Technology 29, 124003 (2016).

9. Y. Braiman, B. Neschke, N. Nair, N. Imam, R. Glowinski, Physical Review E 94, 052223 (2016).

10. S. K. Tolpygo, V. Bolkhovsky, T. J. Weir, L. Johnson, M. Gouker, and W. D. Oliver, IEEE Transactions on Applied Superconductivity 25, 110312 (2015).

11. Corana, M. Marchesi, C. Martini, and S. Ridella (1987), ACM. Trans. Math. Soft. 13, 262 (1987).

12. N. Imam, E.N. Glytsis, T.K. Gaylord, K-K. Choi, P.G. Newman, and L. Detter-Hoskin (2003), IEEE Journal of Quantum Electronics 39, 468 (2003).

13. S. Kirkpatrick, C. D. Gelatt, and M. P. Science, New Series 220 (4598), 671-680 (1983).

14. R. Gross and A. Marx (2015), Lectures on Applied Superconductivity, http://www.wmi.badw.de/teaching/Lecturenotes/index.html 